\title{
Serum N-terminal pro-brain natriuretic peptide as a prognostic marker in patients with hypertrophic cardiomyopathy
}

\author{
Renata Mączyńska-Mazuruk²,2, Łukasz A. Małek³, Mariusz Kłopotowski', Krzysztof Kukuła', Maciej Dąbrowski', \\ Rafał Baranowski', Artur Oręziak¹, Maciej Sterliński', Paweł Syska', Anna Klisiewicz', Zbigniew Chmielak ${ }^{1}$, Adam Witkowski \\ 1 Institute of Cardiology, Warsaw, Poland \\ 2 Midtown Clinical Centre, Warsaw, Poland \\ 3 Faculty of Rehabilitation, Józef Piłsudski University of Physical Education in Warsaw, Warsaw, Poland
}

Correspondence to: Łukasz A. Małek, MD, PhD, Faculty of Rehabilitation, Józef Piłsudski University of Physical Education in Warsaw, ul. Marymoncka 34, 00-968 Warszawa, Poland, phone: +48228340431 email: lukasz.a.malek@gmail.com Received: February 8, 2019. Revision accepted: March 1, 2019. Published online: March 1, 2019. Kardiol Pol. 2019; 77 (5): 571-573 doi:10.5603/KP.a2019.0042 Copyright by Polskie Towarzystwo Kardiologiczne, Warszawa 2019
Introduction Hypertrophic cardiomyopathy (HCM) is a common inherited heart disease oc curring in about 1 in 500 people in the general population. In a majority of cases HCM is caused by mutations in genes encoding sarcomere proteins. The disease leads to disarray of cardiomyocytes with secondary interstitial fibrosis, resulting in myocardial hypertrophy with diastolic and systolic dysfunctions. ${ }^{1}$

B-type natriuretic peptide (BNP) is a polypeptide secreted by the ventricular muscle in response to excessive stretching of cardiomyocytes. Its levels are elevated in patients with heart failure, including those with left ventricular outflow tract obstruction at rest or diastolic dysfunction. ${ }^{2}$ BNP or N-terminal fragment of pro-BNP (NT-proBNP) levels are elevated in patients with $\mathrm{HCM}$ also due to silent myocardial ischemia or an end-stage form of HCM represented by systolic heart failure. ${ }^{3}$ The levels of these peptides correlate with functional class assessed according to the New York Heart Association (NYHA) classification. ${ }^{4}$

Both BNP and NT-proBNP are clinically important diagnostic and prognostic markers of ischemic heart disease and heart failure, but the usefulness of these biomarkers for risk stratification in HCM is unclear. The present investigation was set to determine the prognostic utility of NT-proBNP in patients with HCM.

Methods This was an observational, prospective, single-center cohort study. Participants were enrolled between June 2008 and August
2013 at the Institute of Cardiology, Warsaw, Poland from among stable hospitalized or ambulatory patients. The diagnosis of HCM was based on the presence of unexplained myocardial hypertrophy (in the absence of abnormal loading conditions) assessed as maximal left ventricular wall thickness of $15 \mathrm{~mm}$ or more, or in accordance with the criteria for the diagnosis of familial disease in patients with at least 1 first-degree relative with HCM.

Venous blood samples were obtained from all patients. Plasma NT-proBNP level was measured by 2 -site electrochemiluminescence immunoassay on the Cobas e601 analyzer (Roche Diagnostics, Indianapolis, Indiana, United States). Results were reported in $\mathrm{pg} / \mathrm{ml}$. Normal values for the assay (0.00-125.00 pg/ml) were defined using manufacturer's guidelines.

The primary endpoint consisted of all-cause mortality or heart transplantation. Two secondary endpoints included: 1) unscheduled hospitalization due to deterioration of heart failure, and 2) resuscitated sudden cardiac death (SCD) or appropriate intervention of the implanted cardioverter-defibrillator (ICD). Data on ICD intervention were obtained from device interrogation reports. Statistical analysis was performed using the SPSS package (version 20.0, IBM Corporation, Armonk, New York, United States). The study was approved by the local ethics committee.

Results and discussion The study included 603 patients with HCM (57.5\% men, mean [SD] age, 
44 [17] years). Median plasma NT-proBNP concentration in the whole group was $836.3 \mathrm{pg} / \mathrm{ml}$ (interquartile range [IQR], 289.57-1699.25 pg/ $\mathrm{ml})$. NT-proBNP values were higher in women than in men $(P=0.028)$. For the purpose of this analysis, patients in the second and third tertiles of NT-proBNP concentrations (300-1700 pg/ml and $>1700 \mathrm{pg} / \mathrm{ml}$ ) were compared with those in the first tertile $(<300 \mathrm{pg} / \mathrm{ml})$. Patients in the higher tertiles of NT-proBNP concentrations had worse NYHA functional class. NT-proBNP levels were measured in peripheral blood at baseline. Patients were observed for a mean (SD) of 27 (14) months. Primary endpoint occurred in 14 patients (2.3\%) and included 11 deaths and 3 heart transplantations. Fatal events included 9 deaths due to deterioration of heart failure, 1 SCD, and 1 death due to postoperative complications. Secondary endpoints occurred in: 1) 32 patients (5.4\%) and 2) 23 patients (3.7\%), respectively. The latter included 1 resuscitated SCD and 22 appropriate ICD interventions.

Patients in the higher tertiles of NT-proBNP concentrations had a higher risk of death or heart transplantation (hazard ratio [HR], 5.9; 95\% CI, 2.05-17.04; $P=0.001$ ) and unscheduled hospitalizations due to heart failure (HR, 1.76; 95\% CI, 1.03-3.0, $P=0.037$ ) as determined by the multivariable Cox model. Sudden cardiac death or appropriate ICD intervention was not significantly associated with higher levels of NT-proBNP. There were no primary events and only a few appropriate ICD interventions in those in the lowest tertile of NT-proBNP levels.

Patients with NT-proBNP in the second and third tertiles had worse survival rates in comparison to those in the first tertile of the NT-proBNP concentrations ( $P=0.007$ ) (FIGURE1). They also had an increased risk of deterioration of

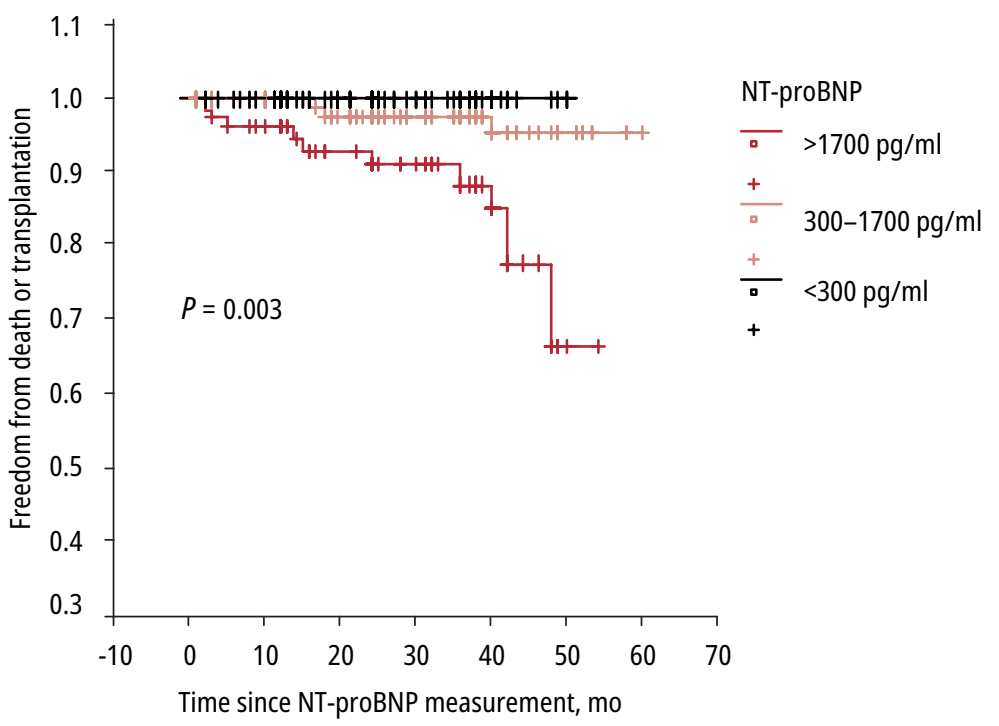

FIGURE 1 Kaplan-Meier curve for the primary endpoint according to tertiles of N-terminal pro-brain natriuretic peptide (NT-proBNP) concentration heart failure followed by an unplanned hospitalization $(P=0.003)$.

NT-proBNP levels below the cutoff point for the detection of heart failure $(<125 \mathrm{pg} / \mathrm{ml})$ were found in 84 patients ( $14 \%$ of the whole group). Mean (SD) age in this group of patients was 31 (11) years. Among those patients there was only a single hospitalization due to exacerbation of heart failure. All of these patients were initially in NYHA class I or II.

We demonstrated that NT-proBNP is an independent predictor of worse clinical outcome in patients with HCM. These results are in line with a previous large prospective study including 847 HCM patients. ${ }^{4}$ Another retrospective analysis on 772 patients showed that higher levels of BNP correlate with all-cause mortality. ${ }^{5}$ Both BNP and NT-proBNP were found to be markers of worse prognosis in few previously published small studies. ${ }^{6-10}$ Our study strongly supports previous findings.

We demonstrated that patients with HCM who were in the lowest tertile of NT-proBNP concentrations, and particularly those with biomarker levels below the cutoff point for heart failure detection ( $<125 \mathrm{pg} / \mathrm{ml})$, have generally good prognosis, as demonstrated by lack of deaths or heart transplantations as well as by a small number of unscheduled hospitalizations in this group of patients. Furthermore, the baseline NYHA class in this group of patients with HCM was also lower than in patients in the higher tertiles of NT-proBNP concentrations. Therefore, these patients can be considered as a low-risk group, provided they are thoroughly screened for the increased risk of SCD and receive an ICD if necessary (we reported 2 appropriate device interventions in this group of patients).

Our study has some limitations. First of all, the results are based on a single NT-proBNP measurement. However, other studies in that area also did not use repetitive biomarker sampling. Secondly, the primary endpoint occurred in a relatively small number of patients. This was mainly influenced by 2 facts: the relatively short period of follow-up and a high frequency of prior ICD implantations in the study group. The device was present at baseline in $28 \%$ of patients in our group and only in $12 \%$ of patients in a previously reported large study. ${ }^{4}$

We found that the NT-proBNP concentration is not a predictor of SCD or appropriate ICD interventions. These data suggest that this biomarker cannot be considered a useful risk factor for better identification of high-risk patients who may benefit from ICD implantation in the primary prevention of SCD.

\section{ARTICLE INFORMATION}

ACKNOWLEDGMENTS The study was funded by the National Science Center (no. 4024699537; to RM-M).

CONFLICT OF INTEREST None declared. 
HOW TO CITE Mączyńska-Mazuruk R, Małek ŁA, Kłopotowski M, et al. Serum $\mathrm{N}$-terminal pro-brain natriuretic peptide as a prognostic marker in patients with hypertrophic cardiomyopathy. Kardiol Pol. 2019; 77: 571-573. doi:10.5603/ KP.a2019.0042

\section{REFERENCES}

1 Maron BJ, Gardin JM, Flack JM, et al. Prevalence of hypertrophic cardiomyopathy in a general population of young adults. Echocardiographic analysis of 4111 subjects in the CARDIA study. Coronary artery risk development in (young) adults. Circulation. 1995; 92: 785-789.

2 Arteaga E, Araujo AQ, Buck P, et al. Plasma aminoterminal pro-B-type natriuretic peptide quantification in hypertrophic cardiomyopathy. Am Heart J. 2005; 150: 1228-1232.

3 Tesic M, Seferovic J, Trifunovic D, et al. N-terminal pro-brain natriuretic peptide is related with coronary flow velocity reserve and diastolic dysfunction in patients with asymmetric hypertrophic cardiomyopathy. J Cardiol. 2017; 70: 323-328.

4 Coats $\mathrm{C}$, Gallagher MJ, Foley M, et al. Relation between serum N-terminal pro-brain natriuretic peptide and prognosis in patients with hypertrophic cardiomyopathy. Eur Heart J. 2013; 34: 2529-2533.

5 Geske JB, McKie PM, Ommen SR, Sorajja P. B-type natriuretic peptide and survival in hypertrophic cardiomyopathy. J Am Coll Cardiol. 2013; 61: 2456-2460.

6 Kubo T, Kitaoka H, Okawa M, et al. Combined measurements of cardiac troponin I and brain natriuretic peptide are useful for predicting adverse outcomes in hypertrophic cardiomyopathy. Circ J. 2011; 75: 919-926.

7 Efthimiadis GK, Hitoglou-Makedou A, Giannakoulas G, et al. Clinical significance of $\mathrm{N}$-terminal-probrain natriuretic peptide in hypertrophic cardiomyopathy. Heart Vessels. 2007; 22: 322-327.

8 D'Amato R, Tomberli B, Castelli G, et al. Prognostic value of N-terminal pro-brain natriuretic peptide in outpatients with hypertrophic cardiomyopathy. Am J Cardiol. 2013; 112: 1190-1196.

9 Mutlu B, Bayrak F, Kahveci G, et al. Usefulness of N-terminal pro-B-type natriuretic peptide to predict clinical course in patients with hypertrophic cardiomyopathy. Am J Cardiol. 2006; 98: 1504-1506.

10 Yuki Miyaji Y, Iwanaga Y, Nakamura T, et al. Interrelationship between the myocardial mass, fibrosis, BNP, and clinical outcomes in hypertrophic cardiomyopathy. Intern Med. 2016; 55: 1261-1268. 